\title{
Microclima en el interior de un bosque de Nothofagus pumilio y el efecto de una corta de regeneración
}

\author{
Microclimate within a Nothofagus pumilio forest and the effects of a regeneration felling
}

\author{
Alvaro Promis ${ }^{a *}$, Juan Caldentey ${ }^{a}$, Manuel Ibarra ${ }^{a}$

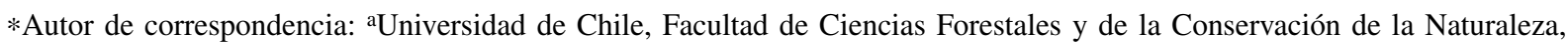 \\ Departamento de Silvicultura y Conservación de la Naturaleza, Santiago, Chile, tel.: 00562978 5721, alvaro.promis@gmail.com
}

\begin{abstract}
SUMMARY
The forest microclimate in a Nothofagus pumilio primary forest $(B V)$ and the effect of a regeneration felling $(R G)$ and an open field $(S D)$ were studied during seven years. Three weather stations measured the global solar radiation $(R S G)$, the air temperature $\left(T_{a}\right)$, the soil temperature at $15\left(T S_{15}\right)$ and $30\left(T S_{30}\right) \mathrm{cm}$ depth, the relative humidity $\left(H R_{a}\right)$ and the wind speed $(V)$. The results showed that the trends in meteorological variables were similar to those found in forests with similar characteristics. Almost all meteorological variables recorded in $B V$ showed statistical differences, compared to $S D$, with the exception of $T_{a}$, and during the leafless period with $T_{\text {mina }}$ (minimum air temperature) $T S_{15}$ and $T S_{30}$. When $C R$ was compared to $B V$ the variables $R S G, H R_{a}, T S_{15}, T S_{30}$ (only during leafless period) and $V$ showed significant differences. The higher differences between $B V$ and $C R$ were that in $C R$ the $R S G$ was 4.4 and 3.5 times higher than in $B V$, for the fully-leaved and the leafless period; and $V$ was 1.2 times higher in $C R$ than in $B V$. These differences suggest that the microclimate in $C R$ changed respect $B V$. Because the microclimate and the water balance in the stand affect the vegetation, the differences found between $B V$ and $C R$ are important, and provide a baseline of information to evaluate the effects of the regeneration felling, the most frequently implemented cut in these forests.
\end{abstract}

Key words: Nothofagus pumilio, forest microclimate, primary forest, regeneration felling, Patagonia.

\section{RESUMEN}

El microclima en un rodal virgen de Nothofagus pumilio $(B V)$ y el efecto de una corta de regeneración $(C R)$ y de un sector desarbolado $(S D)$ fueron estudiados durante siete años. Tres estaciones microclimáticas registraron las variables: radiación solar global $(R S G)$, temperaturas del aire $\left(T_{a}\right)$ y del suelo a $15\left(T S_{15}\right)$ y $30 \mathrm{~cm}\left(T S_{30}\right)$ de profundidad, humedad relativa $\left(H R_{a}\right)$ y velocidad del viento $(V)$. Los resultados mostraron que las tendencias en las variables meteorológicas se ajustaron al comportamiento que éstas normalmente exhiben en bosques de similares características. $B V$ presentó diferencias estadísticamente significativas con $S D$ en todas las variables microclimáticas, excepto con $T_{a}$, y durante los períodos de receso vegetativo con $T_{\text {mina }}, T S_{15}$ y $T S_{30}$. En $C R$ se produjeron cambios estadísticamente significativos respecto a $B V$ en las variables $R S G, H R_{a}, T S_{15}, T S_{30}$ (solamente durante período receso vegetativo) y $V$. Las mayores diferencias encontradas entre $B V$ y $C R$ fueron que $R S G$ aumentó en promedio 4,4 y 3,5 veces en $C R$ durante períodos de crecimiento y receso vegetativo, respectivamente; y que la $V$ aumentó 1,2 veces en $C R$. Las diferencias en las condiciones microclimáticas registradas sugieren que en $C R$ se verifica un microclima distinto al del $B V$. Debido a que el microclima y el balance hídrico en el rodal influyen en la vegetación, las disparidades encontradas entre $B V$ y $C R$ son importantes y entregan una base útil para evaluar las implicancias de la práctica forestal más utilizada en estos bosques.

Palabras clave: Nothofagus pumilio, microclima forestal, bosque virgen, corta de regeneración, Patagonia.

\section{INTRODUCCIÓN}

El microclima define el conjunto de condiciones climáticas propias de un punto geográfico o área reducida y representa una modificación local del clima general de la región debido a la influencia de distintos factores ecológicos (Barnes et al. 1998). La presencia de cubiertas vegetales y particularmente de cubiertas forestales modifica los parámetros climáticos y crea un microclima cuyas particularidades dependen del clima general y de las características estructurales de la cubierta vegetal (Lee 1978, Aussenac 2000). Debido a la alta acumulación de biomasa y a las dimensiones de los árboles, los bosques tienen una considerable influencia en los intercambios de energía entre la atmósfera y el suelo, actuando la cubierta vegetal sobre el comportamiento de la radiación solar, las precipitaciones, la humedad atmosférica, la temperatura del aire, el viento y la capacidad evaporativa del aire (Aussenac 1997, Barnes et al. 1998, Geiger et al. 2003). El crecimiento de las plantas se ve afectado por la temperatura del aire, la radiación solar y la humedad debido a que estos elementos influyen sobre procesos fisiológicos tales como la fotosíntesis, la respiración, la germinación de semillas y la mortalidad (Barnes et al. 1998). 
Tanto las perturbaciones naturales como también las antropogénicas, al alterar las características estructurales de las cubiertas vegetales, modifican el ambiente físico de un ecosistema afectando su productividad, diversidad biológica y patrones sucesionales (Lee 1978, Brosofske et al. 1997, Chen et al. 1999, Decocq et al. 2005).

La composición y la estructura de los bosques son comúnmente manipuladas mediante la aplicación de tratamientos silviculturales, con el propósito de producir madera y otros productos forestales. En general se trata de mejorar el crecimiento de árboles seleccionados y de asegurar su regeneración (Crow et al. 2002, Nauertz et al. 2004). Las prácticas silviculturales causan la remoción parcial o total del dosel arbóreo alterando las propiedades térmicas superficiales y los balances energéticos cerca del nivel del suelo, con el consecuente cambio en el microclima dentro del bosque (Lee 1978, Chen et al. 1993, Aussenac 1997, 2000, Brosofske et al. 1997, Carlson y Groot 1997, Langvall y Örlander 2001, Proe et al. 2001, Geiger et al. 2003, Nauertz et al. 2004).

Nothofagus pumilio (Poepp. et Endl.) Krasser, conocida como lenga, es una especie caducifolia y la más característica de los bosques de la Patagonia chilena y del archipiélago de Tierra del Fuego. El tipo forestal lenga cubre una superficie aproximada de 1.125.000 ha en la región de Magallanes (CONAF et al. 1999), y es el principal producto comercial maderero en el sur de Chile y Argentina (Schmidt 1994, Martínez Pastur et al. 2000, Gea-Izquierdo et al. 2004). El sistema silvicultural que ha resultado más adecuado para intervenir los bosques de lenga con potencial comercial es el de cortas sucesivas con cortas de regeneración natural bajo dosel de protección o también llamado cortas de protección (Schmidt et al. 2003). En bosques vírgenes maduros el sistema se inicia con cortas de regeneración que disminuyen la cobertura del dosel arbóreo entre el 40 y el $60 \%$, con ello aumentan los montos de luminosidad, favoreciendo el establecimiento de la regeneración y su crecimiento en altura (Schmidt et al. 2003, Rosenfeld et al. 2006, Caldentey et al. 2009).

Los impactos del tratamiento silvicultural en la regeneración de $N$. pumilio y en la estructura del bosque durante el ciclo completo del sistema silvicultural han sido bastante estudiados (Schmidt y Urzúa 1982, Schmidt 1994, Bartsch y Rapp 1995, Loguercio 1995, Martínez Pastur et al. 1999, 2000, Schmidt et al. 2003, Rosenfeld et al. 2006). Se han comprobado cambios en la diversidad de especies que conforman el sotobosque (Martínez Pastur et al. 2002), en la diversidad de insectos (Spagarino et al. 2001) y de pájaros (Venegas y Schlatter 1999, Deferrari et al. 2001, Lencinas et al. 2005, Vergara y Schlatter 2006), y en los aportes y las tasas de descomposición e incorporación de residuos de hojarasca al piso de los bosques (Caldentey et al. 2001).

Respecto a cambios microclimáticos en estos bosques debido a la aplicación de cortas de regeneración, solamente se han delineado tendencias generales a partir de un tiempo reducido de observaciones (Caldentey et al. 1999, 2000), registrándose además aumentos en el flujo de fotones fotosintéticos (Caldentey et al. 2009). En este estudio el objetivo es analizar el comportamiento de variables microclimáticas a lo largo de siete años de registros continuos, en un bosque de $N$. pumilio no intervenido, en un bosque sometido a una corta de regeneración y en un sector desarbolado. De esta manera se entrega información base para el estudio de los efectos de los tratamientos silviculturales en la composición, estructura, y función de bosques de $N$. pumilio en la región de Magallanes.

\section{MÉTODOS}

Área de estudio. La investigación se realizó en la localidad de Monte Alto ubicada en las coordenadas $52^{\circ} 08^{\prime} \mathrm{S}, 71^{\circ}$ 38' O, a una elevación de $460 \mathrm{~m}$ s.n.m. (figura 1). El sector estudiado presenta un clima templado frío constantemente húmedo (Cf). La zona se inserta en el distrito agroclimático de Punta Arenas, que presenta una temperatura mínima media del mes más frío (julio) de $-9,3^{\circ} \mathrm{C}$ y una temperatura máxima media del mes más cálido (enero) de $15,3^{\circ} \mathrm{C}$. La velocidad del viento normalmente es muy alta en los meses de primavera y verano, pudiendo alcanzar velocidades de $120 \mathrm{~km} / \mathrm{h}$ en noviembre (INIA 1989).

Fisiográficamente, el estudio se desarrolló en una faja transicional entre la Cordillera de Los Andes y las planicies patagónicas orientales. Es un terreno mesetiforme de baja altura, atravesado por cordones montañosos bajos, y fuertemente modificado por fenómenos glaciales (Pisano 1977). Los suelos clasificados como podzólicos y grises de bosques (Pisano 1981) son originados in situ. Tienen profundidades bajas a medias, presentan texturas livianas y medias, estructuras granulares en superficie y de bloques

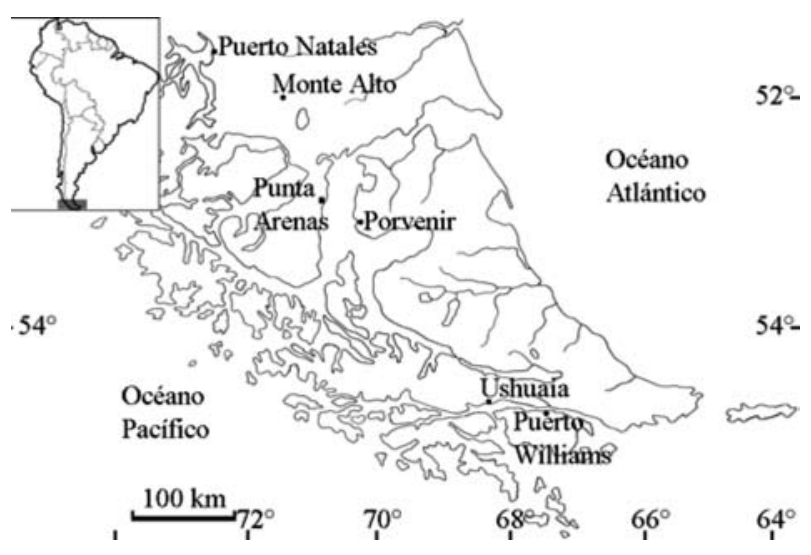

Figura 1. Mapa de Sudamérica, la Patagonia Austral y Tierra del Fuego mostrando la ubicación del área de estudio en Monte Alto.

Map of South America, southern Patagonia and Tierra del Fuego, showing the study area location at Monte Alto. 
subangulares en profundidad, $\mathrm{pH}$ ácidos y bajos contenidos de materia orgánica. El arraigamiento de los árboles se concentra en los primeros $40 \mathrm{~cm}$ de profundidad y no se presenta erosión bajo las cubiertas forestales (Contreras et al. 1975).

La formación vegetal característica de esta zona es la denominada bosque caducifolio de Magallanes (Gajardo 1994) cuya única especie dominante arbórea es $N$. pumilio, con un estrato arbustivo dominado por Berberis buxifolia Lam., Maytenus disticha (Hooker f.) Urban, Chilotrichum diffusum (Forster f.) Kuntze, Pernettya mucronata (L. f.) Gaudich ex Don, y una cobertura herbácea representada por Acaena ovalifolia Ruiz et Pavón, Blechnum pennamarina (Poiret) Kuhn, Gunnera magallanica Lam y Osmorhiza chilensis Hooker et Arn. (Skottsberg 1910, Roig et al. 1985, Lara y Cruz 1987, Gajardo 1994).

Selección de superficies de ensayo. Se definieron tres situaciones de estudio, dos cubiertas con bosques de $N$. pumilio y otra en un terreno descubierto $(S D)$. Los sectores boscosos analizados, de 2 ha cada uno, fueron un bosque virgen $(B V)$, el cual no presentaba evidencias de intervenciones antrópicas en el pasado, y un rodal sometido a una corta de regeneración $(C R)$ en el año 1994, con fines de producción forestal. La altura promedio de los árboles dominantes en ambos rodales fue de $21 \mathrm{~m}$. El rodal virgen estaba constituido por árboles en fases de desarrollo de envejecimiento y desmoronamiento de acuerdo a Schmidt y Urzúa (1982), con una densidad media de $890 \pm 40$ árboles/ha y un área basal promedio de $80 \pm 5,1 \mathrm{~m}^{2} / \mathrm{ha}$. La corta de regeneración se aplicó a un rodal con existencias totales originales semejantes al rodal virgen (Schmidt et al. 2003) y se enmarcó en las regulaciones técnicas legales de manejo silvicultural sugeridas para este tratamiento y tipo forestal (Rosenfeld et al. 2006). Al momento de la instalación del ensayo, después de la corta de regeneración, el rodal intervenido presentaba una densidad de $213 \pm 57$ árboles/ha y un área basal de $36 \pm 7,0 \mathrm{~m}^{2} / \mathrm{ha}$.

Instrumental meteorológico. En cada uno de los rodales y en el sector desarbolado se instaló una estación agroclimática automática. Éstas se encontraban equipadas con módulos de almacenamiento de datos CAMPBELL Scientific MR10, conectadas a paneles solares y baterías de respaldo, en las que se registraron las siguientes variables microclimáticas: I.- Radiación solar global (cal/ $\mathrm{cm}^{2}$ ) medida con tres piranómetros LI-COR LI 200SA a dos metros sobre el nivel del suelo, de los cuales se instalaron tres en cada uno de los rodales y uno en el sector desarbolado; II.- Temperatura del aire $\left({ }^{\circ} \mathrm{C}\right)$ y humedad relativa del aire (\%) registradas con sondas CAMPBELL CS500U, con un protector de radiación no aspirado, a tres metros sobre el suelo; III.- Temperatura del suelo $\left({ }^{\circ} \mathrm{C}\right)$ medida con sondas Campbell 107B a 15 y $30 \mathrm{~cm}$ de profundidad; IV.- Velocidad del viento $(\mathrm{km} / \mathrm{h})$ registrada mediante un anemómetro de cazoletas
Climatronics WM-III P/N 100108-1, ubicado a una altura que correspondía a la mitad del dosel de copas en los rodales, y en el sector desarbolado a $4 \mathrm{~m}$ sobre el nivel del suelo.

Mediciones. Las mediciones se realizaron desde marzo 1997 hasta marzo 2004 (siete años). Las estaciones meteorológicas efectuaron registros cada 60 segundos de las variables microclimáticas y calcularon valores horarios de cada una de ellas. Para completar eventuales valores no compilados se desarrollaron ecuaciones de regresión para cada una de las variables microclimáticas $\left(\mathrm{R}^{2}>0,81 ; P<0,01\right)$, semejante a lo señalado por Nauertz et al. (2004).

Se determinaron promedios mensuales y por períodos de crecimiento (octubre-marzo) y receso vegetativo (abrilseptiembre) de los parámetros: radiación solar global $(R S G)$, temperatura media del aire $\left(T_{a}\right)$, temperatura media mínima del aire $\left(T_{\text {mina }}\right)$, temperatura media máxima del aire $\left(T_{\text {maxa }}\right)$, humedad relativa media del aire $\left(H R_{a}\right)$, temperaturas media del suelo a 15 y $30 \mathrm{~cm}$ de profundidad $\left(T S_{15}, T S_{30}\right)$, y velocidades media mensual (V) y media extrema $\left(V_{e}\right)$ del viento.

Se realizaron comparaciones estadísticas entre los tratamientos y los registros micrometeorológicos para cada uno de los siete períodos de crecimiento y receso vegetativo, por medio de la prueba no paramétrica de comparaciones de pares Mann-Whitney U. Todos los análisis estadísticos fueron desarrollados utilizando SPSS 15.0 para Windows (SPSS Inc.).

\section{RESULTADOS}

Microclima en el bosque de N. pumilio. A través de todo el año se pudo observar que la $R S G$ medida dentro del $B V$ es más reducida que aquella registrada en el $S D$ (figura 2). Durante el período de crecimiento vegetativo, la acumulación promedio mensual de radiación en el bosque fue de $1.114 \mathrm{cal} / \mathrm{cm}^{2}$, cerca de un $12,6 \%$ del promedio mensual incidente en la estación $S D$, con diferencias estadísticas significativas entre ambas situaciones $(P<0,01)$. En el período de receso vegetativo, el valor promedio de la $R S G$ fue de sólo $372 \mathrm{cal} / \mathrm{cm}^{2}$ en el interior del bosque, cerca del $14,1 \%$ de los registrados en el $S D$, presentando también diferencias estadísticas $(P<0,01)$ (cuadro 1).

La $T_{a}$ en el bosque virgen mostró un patrón similar al registrado en el $S D$ (figura 3). La $T_{a}$ no arrojó diferencias estadísticas entre ambos sectores, para ninguno de los períodos de crecimiento y receso vegetativo $(P=0,259$ y $P=0,534$, respectivamente). En cambio, la $T_{\operatorname{maxa}}$ siempre fue estadísticamente menor dentro del bosque al compararla con el $S D$, con diferencias de $0,9^{\circ} \mathrm{C}$ y $1,3^{\circ} \mathrm{C}$ para los promedios registrados durante los períodos de crecimiento y receso vegetativo, respectivamente $(P<0,05)$. La $T_{\text {mina }}$ del aire en el $B V$ siempre fue más elevada que fuera del bosque, pero solamente en el período de crecimiento vegetativo estas diferencias fueron estadísticamente significativas 
Cuadro 1. Radiación solar global promedio $(R S G)$, temperatura media del aire $\left(T_{a}\right)$, temperatura media mínima del aire $\left(T_{\text {mina }}\right)$, temperatura media máxima del aire $\left(T_{\text {maxa }}\right)$, humedad relativa del aire $\left(H R_{a}\right)$, temperaturas media del suelo a $15 \mathrm{~cm}$ y a $30 \mathrm{~cm}$ de profundidad $\left(T S_{15}\right.$ y $\left.T S_{30}\right)$, velocidades media del viento $(V)$ y extrema del viento $\left(V_{e}\right)$, para dos rodales de $N$. pumilio $(B V, C R)$ y un sector desarbolado, durante los períodos de crecimiento vegetativo (octubre-marzo) y receso vegetativo (abril-septiembre). Igualdad de letras significa que no existe diferencia estadística significativa entre tratamientos (Mann-Whitney $\mathrm{U}, P>0,05$ ). Medias \pm desviaciones estándares.

Mean monthly global solar radiation $(R S G)$, mean monthly air temperature $\left(T_{a}\right)$, mean monthly minimum air temperature $\left(T_{\text {mina }}\right)$, mean monthly maximum air temperature $\left(T_{\text {maxa }}\right)$, mean relative humidity $\left(H R_{a}\right)$, mean monthly soil temperature at 15 and $30 \mathrm{~cm}$ depth $\left(T S_{15}, T S_{30}\right)$, mean monthly wind speed $(V)$ and mean monthly peak wind speed $\left(V_{e}\right)$, within two lenga stands $(B V, C R)$ and in an open field $(S D)$, for the fully-leaved period (October- March) and the leafless period (April-September). Similar letters mean no significant differences among treatments (Mann-Whitney $\mathrm{U}$-Test, $P>0.05)$. Numbers in parenthesis are standard deviations.

\begin{tabular}{llccc}
\hline Variables & Períodos & SD & BV & CR \\
\hline$R S G\left(\mathrm{cal} / \mathrm{cm}^{2} / \mathrm{mes}\right)$ & Crecimiento & $8.805 \pm 382 \mathrm{a}$ & $1.114 \pm 59 \mathrm{~b}$ & $4.637 \pm 125 \mathrm{c}$ \\
& Receso & $2.629 \pm 301 \mathrm{a}$ & $372 \pm 35 \mathrm{~b}$ & $1.296 \pm 59 \mathrm{c}$ \\
$T_{a}\left({ }^{\circ} \mathrm{C}\right)$ & Crecimiento & $7,0 \pm 0,5 \mathrm{a}$ & $6,7 \pm 0,6 \mathrm{a}$ & $6,8 \pm 0,5 \mathrm{a}$ \\
& Receso & $1,4 \pm 0,8 \mathrm{a}$ & $1,1 \pm 0,8 \mathrm{a}$ & $1,6 \pm 0,8 \mathrm{a}$ \\
$T_{\text {mina }}\left({ }^{\circ} \mathrm{C}\right)$ & Crecimiento & $2,2 \pm 0,3 \mathrm{a}$ & $2,9 \pm 0,5 \mathrm{~b}$ & $2,8 \pm 0,4 \mathrm{~b}$ \\
& Receso & $-2,5 \pm 0,9 \mathrm{a}$ & $-1,6 \pm 0,9 \mathrm{a}$ & $-1,2 \pm 0,8 \mathrm{a}$ \\
$T_{\text {maxa }}\left({ }^{\circ} \mathrm{C}\right)$ & Crecimiento & $11,9 \pm 0,9 \mathrm{a}$ & $11,0 \pm 0,9 \mathrm{~b}$ & $11,8 \pm 0,9 \mathrm{ab}$ \\
& Receso & $5,4 \pm 0,9 \mathrm{a}$ & $4,1 \pm 0,9 \mathrm{~b}$ & $4,8 \pm 0,9 \mathrm{ab}$ \\
$H R_{a}(\%)$ & Crecimiento & $80 \pm 2 \mathrm{a}$ & $84 \pm 3 \mathrm{~b}$ & $79 \pm 3 \mathrm{a}$ \\
& Receso & $90 \pm 1 \mathrm{a}$ & $93 \pm 2 \mathrm{~b}$ & $88 \pm 2 \mathrm{a}$ \\
$T S_{15}\left({ }^{\circ} \mathrm{C}\right)$ & Crecimiento & $7,4 \pm 0,5 \mathrm{a}$ & $5,6 \pm 0,5 \mathrm{~b}$ & $6,4 \pm 0,7 \mathrm{~b}$ \\
& Receso & $1,5 \pm 0,4 \mathrm{a}$ & $1,8 \pm 0,4 \mathrm{a}$ & $1,7 \pm 0,3 \mathrm{a}$ \\
$T S_{30}\left({ }^{\circ} \mathrm{C}\right)$ & Crecimiento & $7,4 \pm 0,4 \mathrm{a}$ & $5,5 \pm 0,5 \mathrm{~b}$ & $6,3 \pm 0,7 \mathrm{~b}$ \\
& Receso & $3,0 \pm 1,0 \mathrm{a}$ & $2,6 \pm 0,5 \mathrm{a}$ & $2,1 \pm 0,3 \mathrm{~b}$ \\
$V(\mathrm{~km} / \mathrm{h})$ & Crecimiento & $11,8 \pm 0,8 \mathrm{a}$ & $2,9 \pm 0,2 \mathrm{~b}$ & $7,1 \pm 0,4 \mathrm{c}$ \\
$V_{e}(\mathrm{~km} / \mathrm{h})$ & Receso & $16,8 \pm 1,3 \mathrm{a}$ & $3,4 \pm 0,3 \mathrm{~b}$ & $4,1 \pm 0,4 \mathrm{c}$ \\
& Crecimiento & $99,7 \pm 5,5 \mathrm{a}$ & $40,4 \pm 6,2 \mathrm{~b}$ & $47,0 \pm 2,5 \mathrm{~b}$ \\
& Receso & $90,7 \pm 11,2 \mathrm{a}$ & $40,2 \pm 7,2 \mathrm{~b}$ & $4,1 \mathrm{~b}$ \\
\hline
\end{tabular}

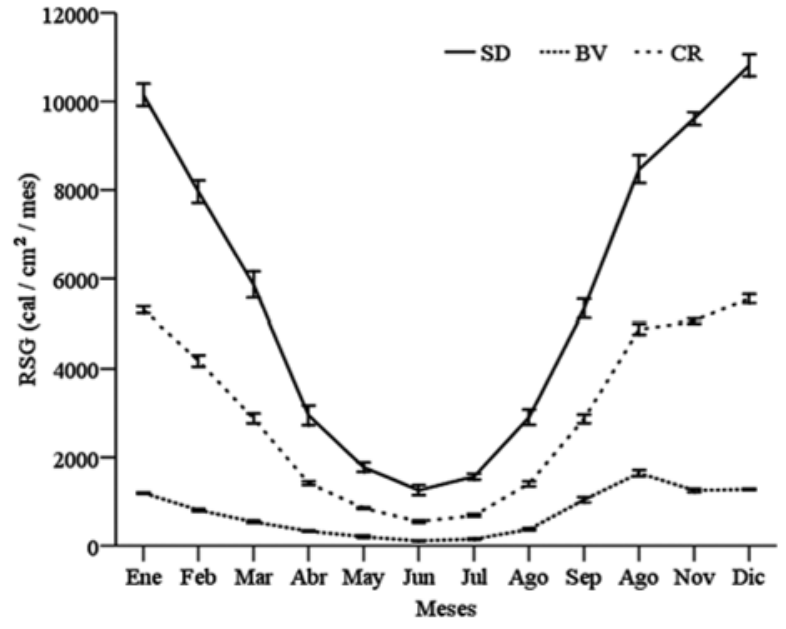

Figura 2. Radiación solar global promedio mensual $(R S G)$ en dos rodales de lenga $(B V, C R)$ y un sector desarbolado $(S D)$. Barras representan el error estándar.

Mean monthly global solar radiation $(R S G)$ within two lenga stands $(B V, C R)$ and in an open field $(S D)$. Error bars represent SEs.
$(P<0,05$ y $P=0,073$ para los períodos de crecimiento y receso vegetativo) (figura $3 \mathrm{y}$ cuadro 1 ).

Los patrones de $H R_{a}$ siempre fueron mayores en el $B V$ (figura 4) en comparación con el $S D$ y mostraron diferencias significativas para los períodos de crecimiento y receso vegetativo ( $P<0,05$ y $P<0,01$, respectivamente) (cuadro 1 ).

Tanto en el $B V$ como en el $S D$, las temperaturas medias del suelo durante el período estival tendieron a disminuir en profundidad, observándose una tendencia inversa en invierno, cuando éstas fueron menores a menor profundidad (figura 5). La $T S_{15}$ en el $B V$ fue significantemente inferior en $1,8^{\circ} \mathrm{C}$ durante el período de crecimiento $(P<0,01)$. Al contrario, durante el período de receso esta variable fue mayor en $0,3^{\circ} \mathrm{C}$, sin mostrar diferencias estadísticas $(P=0,209)$ (cuadro 1). La $T S_{30}$ muestra una tendencia semejante a la anterior, durante el período de crecimiento el $B V$ tuvo en promedio una $T S_{30}$ significativamente menor en $1,9^{\circ} \mathrm{C}$ con respecto al SD $(P<0,01)$. Durante el receso vegetativo fue $0,4^{\circ} \mathrm{C}$ menor, pero estadísticamente similar $(P=0,456)$ (cuadro 1$)$. 
En ambos sectores, la $V$ fue mayor durante el período estival, siendo siempre más alta en el $S D$ (figura 6). En el $B V$ se redujo significativamente la $V$ media $(P<0,01)$

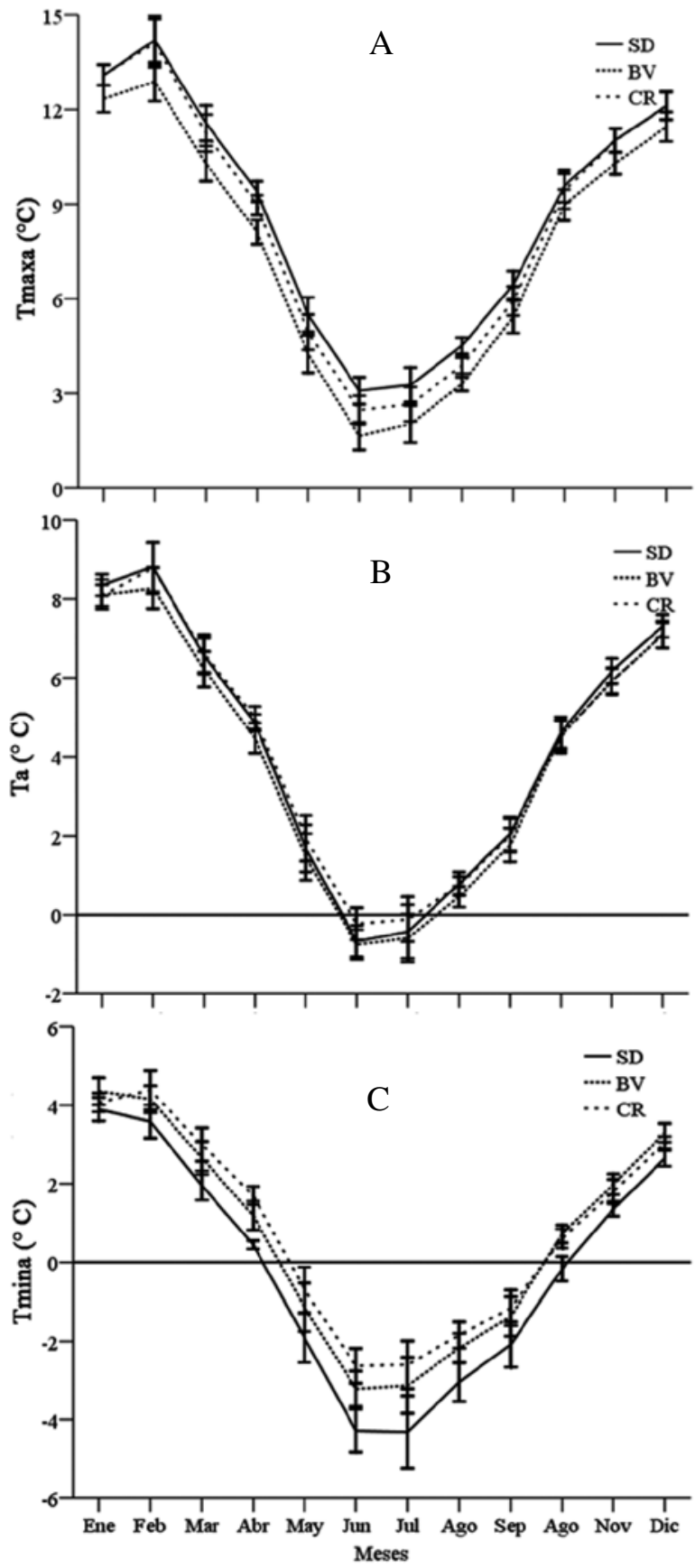

Figura 3. Temperaturas A) media máxima mensual del aire $\left(T_{\text {maxa }}\right)$, B) media mensual $\left(T_{a}\right)$ y C) media mínima mensual $\left(T_{\text {mina }}\right)$ en dos rodales de lenga $(B V, C R)$ y un sector desarbolado $(S D)$. Barras representan el error estándar.

A) Mean monthly maximum air temperature regime $\left(T_{\text {maxa }}\right)$, B) mean monthly air temperature $\left(T_{a}\right)$ and C) mean monthly minimum air temperature $\left(T_{\text {mina }}\right)$ within two lenga stands $(B V, C R)$ and in an open field $(S D)$. Error bars represent SEs.

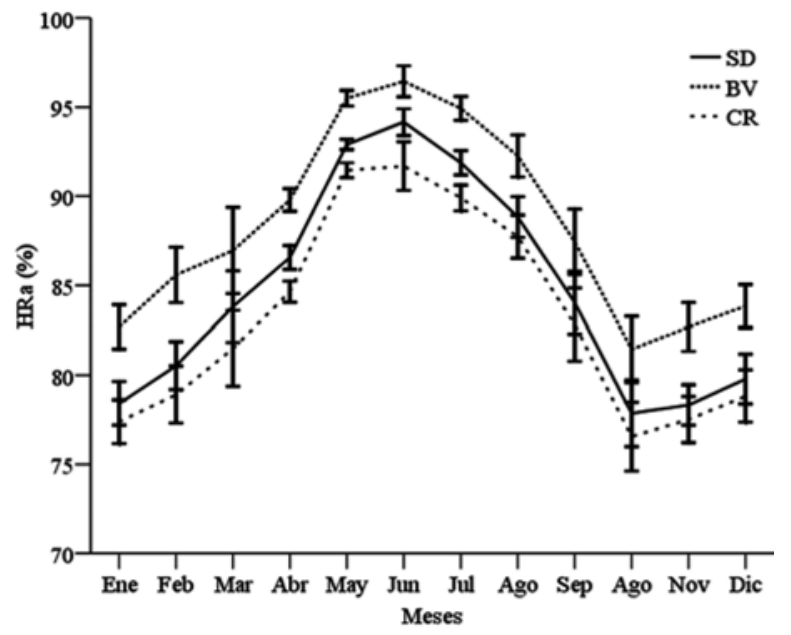

Figura 4. Humedad relativa media del aire $\left(H R_{a}\right)$ en 2 rodales de lenga $(B V, C R)$ y un sector desarbolado $(S D)$. Barras representan el error estándar.

Mean relative humidity $\left(H R_{a}\right)$ within two lenga stands $(B V$, $C R)$ and in an open field $(S D)$. Error bars represent SEs.

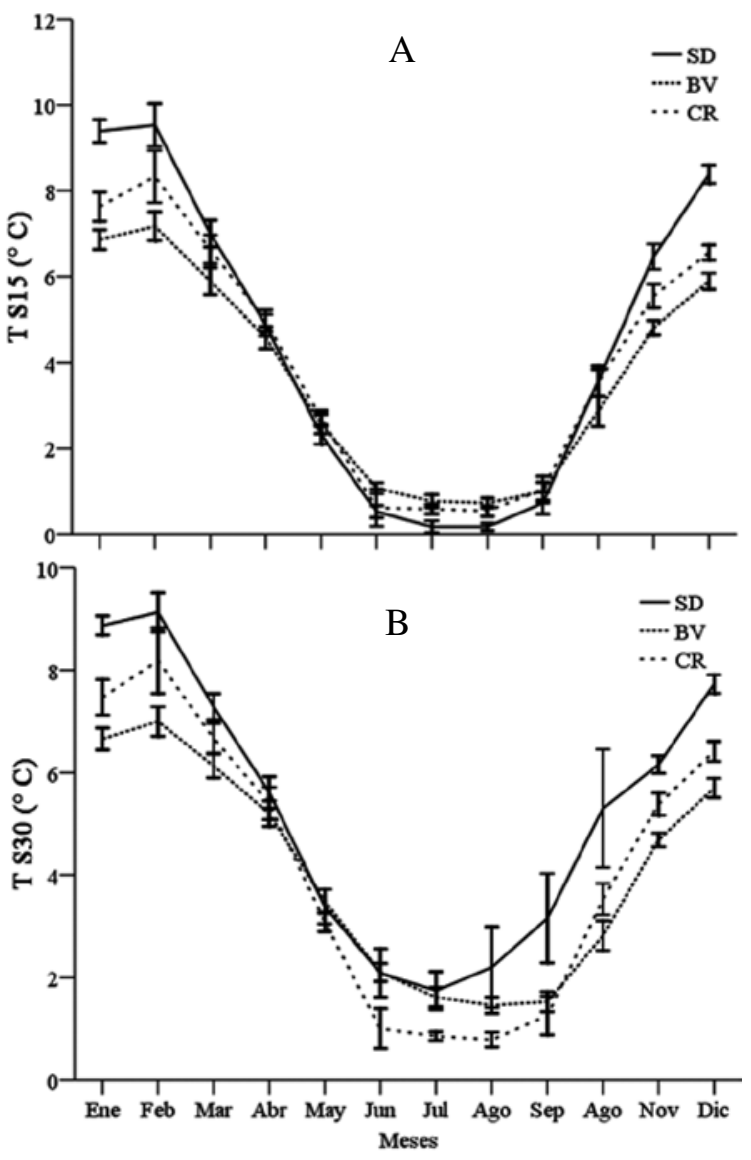

Figura 5. Temperaturas A) media del suelo a 15 y B) $30 \mathrm{~cm} \mathrm{de}$ profundidad $\left(T S_{15}\right.$ y $\left.T S_{30}\right)$ en dos rodales de lenga $(B V, C R)$ y un sector desarbolado $(S D)$. Barras representan el error estándar.

Mean monthly soil temperatures at A) 15 and B) $30 \mathrm{~cm}$ depth $\left(T S_{15}\right.$ y $\left.T S_{30}\right)$ within two lenga stands $(B V, C R)$ and in an open field $(S D)$. Error bars represent SEs. 


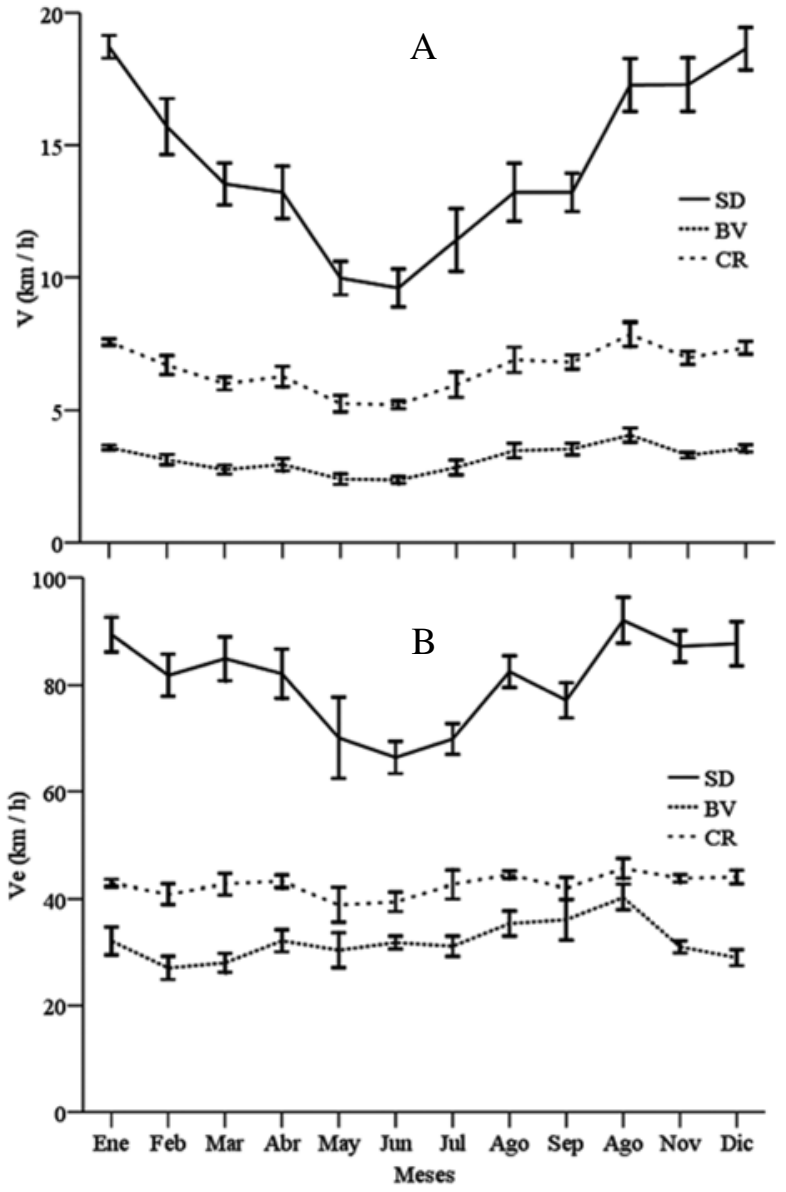

Figura 6. A) Velocidad media mensual del viento $(V)$ y B) velocidad media de las extremas del viento $\left(V_{e}\right)$ en dos rodales de lenga $(B V, C R)$ y un sector desarbolado $(S D)$. Barras representan el error estándar.

A) Mean monthly wind speed $(V)$ and B) mean monthly peak wind speed $\left(V_{e}\right)$ within two lenga stands $(B V, C R)$ and in an open field $(S D)$. Error bars represent SEs.

durante el período de crecimiento vegetativo en cerca del $25 \%$ al compararla con el $S D$. En el período de receso vegetativo, el valor promedio de $V$ fue de $3,4 \mathrm{~km} / \mathrm{h}$ en el interior del $B V$, monto que sólo representó cerca del $20 \%$ de lo registrado en $S D$, presentándose diferencias estadísticas significativas $(P<0,01)$ (cuadro 1$)$.

La $V_{e}$ fue estadísticamente más elevada en el $S D$ que en el $B V(P<0,01)$ (figura 6). Los valores máximos se verificaron en promedio durante los períodos primaverales (septiembre-octubre). En el $B V$ las intensidades de las ráfagas de viento fueron entre 41 y $44 \%$ de las velocidades medias extremas del viento registradas en el $S D$ para los períodos de crecimiento y receso vegetativo (cuadro 1). Además, la máxima velocidad del viento registrada en $S D$ fue de $109,0 \mathrm{~km} / \mathrm{h}$ (26 de octubre de 2003) y la observada en $B V$ fue de solamente $52,8 \mathrm{~km} / \mathrm{h}$ (12 de septiembre de 1999).
Influencia microclimática de una corta de regeneración en el bosque de N. pumilio. La $R S G$ en $C R$ presentó una clara tendencia intermedia entre aquellos registrados en el $S D$ y el $B V$ (figura 2). Los valores medios para los períodos de crecimiento y de receso vegetativo fueron estadísticamente superiores en la $C R$ que en el $B V(P<0,01)$. En el $C R$, durante el período de crecimiento vegetativo, la acumulación de radiación global mensual alcanzó un promedio de $4.637 \mathrm{cal} / \mathrm{cm}^{2}$, siendo 4,2 veces más alta que la acumulada en el interior del $B V$. Esta situación se repitió durante el período de receso del crecimiento con valores promedios mensuales de $1.296 \mathrm{cal} / \mathrm{cm}^{2}$ en el sector $C R$, siendo ésta 3,5 veces mayor que la verificada en el $B V$ (cuadro 1).

La $T_{a}$ en $C R$ mostró regularmente un patrón similar al registrado en el interior del $B V$ (figura 3). Los registros de $T_{a}$ no entregaron diferencias estadísticas entre ambas situaciones boscosas $(B V$ y $C R)$ para ninguno de los períodos de crecimiento y receso vegetativo $(P=0,535$ y $P=0,259$, respectivamente), con diferencias de $0,1^{\circ} \mathrm{C}$ durante el período de cobertura foliar y de $0,5^{\circ} \mathrm{C}$ para el período sin hojas (cuadro 1). La $T_{\text {maxa }}$ siempre mostró un comportamiento promedio mayor en $C R$ sin diferencias estadísticas respecto al $B V(P=0,073$ durante período crecimiento y $P=0,165$ para el período receso), y con diferencias de $0,8^{\circ} \mathrm{C}$ y $0,7^{\circ} \mathrm{C}$, respectivamente (cuadro 1 ). La $T_{\text {mina }}$ mostró un comportamiento similar a lo señalado anteriormente y no se presentaron diferencias estadísticas en sus valores promedio para los períodos de crecimiento y receso vegetativo $(P=1,000$ y $P=0,209)$; siendo en promedio $0,1^{\circ} \mathrm{C}$ mayor en el $B V$ que en $C R$ durante el período con follaje, y $0,4^{\circ} \mathrm{C}$ menor en la etapa sin hojas (cuadro 1).

Respecto a $H R_{a}$, los registros en el interior del $B V$ siempre fueron significativamente mayores que en el interior de $C R(P<0,01$ para ambos períodos), con montos promedios de $5 \%$ más altos en ambos períodos del año (figura 4 y cuadro 1 ).

En el $C R$, las temperaturas medias del suelo, al igual que en el $B V$ y en el $S D$, también tienden a disminuir en profundidad durante los períodos estivales, situación que se revirtió durante el invierno, período en que la capa superficial del suelo tendió a ser más fría (figura 5). Al comparar la temperatura media del suelo a los $15 \mathrm{~cm}$ de profundidad entre el $C R$ y el $B V$, no se produjeron diferencias estadísticas significativas, siendo en promedio $0,8^{\circ} \mathrm{C}$ más alta en $C R$ durante el período de crecimiento vegetativo y $1,0^{\circ} \mathrm{C}$ menor durante el receso $(P=0,073$ y $P=0,902$, respectivamente) (cuadro 1). En profundidad, a $30 \mathrm{~cm}$ y durante el período de crecimiento vegetativo, la temperatura del suelo entre los tratamientos presentó una conducta semejante a la anterior, con un registro promedio mayor y no significativo en $C R$ de $0,8^{\circ} \mathrm{C}(P>0,073)$. En cambio, durante los períodos sin follaje la temperatura a $30 \mathrm{~cm}$ de profundidad en $C R$ disminuyó significativamente en comparación con el $B V(P<0,05)$, verificándose una diferencia promedio de $0,5^{\circ} \mathrm{C}$ (cuadro 1 ). 
La $V$ a la altura media de las copas de los árboles reveló leves aumentos de intensidad durante los períodos primavera-verano en ambos rodales, siendo siempre mayores en $C R$ que en $B V$ (figura 6). En ambos períodos de crecimiento y receso vegetativo, la velocidad del viento fue 2,1 veces significativamente más alta en $C R(P<0,01)$ (cuadro 1).

Las ráfagas de viento $\left(V_{e}\right)$ no mostraron diferencias estadísticas entre ambos rodales $(C R$ y $B V)(P=0,097$ durante el crecimiento vegetativo y $P=0,053$ en el receso vegetativo), y aunque la abertura del bosque por medio de la $C R$ presentó en promedio mayores registros de $V_{e}$, éstos fueron solamente 1,2 veces mayores que en el $B V$ durante ambos períodos de crecimiento (figura 6 y cuadro 1 ). Esto además se vio reflejado en que la velocidad máxima del viento registrada en $B V$ alcanzó una intensidad de $52,8 \mathrm{~km} / \mathrm{h}$ (12 de septiembre de 1999) y en la $C R$ de $52,9 \mathrm{~km} / \mathrm{h}(26$ de octubre de 2003).

\section{DISCUSIÓN}

Influencia microclimática del bosque de $\mathrm{N}$. pumilio. Los resultados obtenidos concuerdan con los antecedentes generales señalados por Lee (1978), Aussenac (2000) y Geiger et al. (2003), relativos a la influencia de una cubierta forestal en las modificaciones de los componentes microclimáticos. El bosque en general es más sombrío, menos ventoso, más frío y más húmedo que fuera de él. Este comportamiento ha sido observado tanto en coníferas como también en latifoliadas, y además en bosques ubicados en diversas latitudes (Ghuman y Lal 1987, Chen et al. 1993, Davies-Colley et al. 2000).

La $R S G$ registrada dentro del bosque correspondió a entre un 12 y un $14 \%$ de la medida en el exterior (cuadro 1). Este rango es consistente con observaciones hechas en otros estudios, en que la $R S G$ oscilaría entre $1-2 \%$ y $62 \%$ dependiendo de la cubierta forestal, la estructura del bosque, el índice de área foliar, y la estación del año (Reifsnyder et al. 1971/1972, Ghuman y Lal 1987, Chen et al. 1993, Aussenac 1997, 2000, Carlson y Groot 1997, Davies-Colley et al. 2000, Holst y Mayer 2005).

La $T_{a}$ medida dentro del BV fue semejante a las registradas en otros bosques de N. pumilio. En la zona de Río Tranquilo la $T_{a}$ fue en promedio $-0,4^{\circ} \mathrm{C}$ en invierno y $10,5^{\circ} \mathrm{C}$ (Doberti y Ruz 1981), mientras que en Tierra del Fuego se han registrado valores promedio de $0^{\circ} \mathrm{C}$ en invierno y $10^{\circ} \mathrm{C}$ en verano (Frangi y Ritcher 1994). La variación promedio de temperatura del aire entre el $B V$ y el $S D$ es similar a los $0,61^{\circ} \mathrm{C}$ registrados por Chen et al. (1993) entre un bosque de Tsuga heterophylla (Raf.) Sarg, y una tala rasa adyacente al sur de Washington en Estados Unidos $\left(45^{\circ} 48^{\prime} \mathrm{N}, 121^{\circ} 55^{\prime} \mathrm{O}\right)$, y a $\operatorname{los} 0,7^{\circ} \mathrm{C}$ medidos por Carlson y Groot (1997) en un bosque de Populus tremuloides Michx. en Ontario, Canadá (47 $43^{\prime} \mathrm{N}$, $\left.83^{\circ} 11^{\prime} \mathrm{O}\right)$.
Respecto a la $H R_{a}$, se comprueba una alta interdependencia entre los contenidos de humedad y la temperatura del aire. En las épocas estivales la $H R_{a}$ disminuyó, resultado del aumento en la capacidad de contención de vapor de agua por parte del aire; en tanto, en los inviernos ocurre lo contrario, la $H R_{a}$ aumenta producto de la disminución de la temperatura del aire. Así en general se cumplen las tendencias señaladas por Lee (1978) y Aussenac (2000), respecto a que los bosques son relativamente más húmedos que el exterior. Las diferencias en $H R_{a}$ entre $B V$ y $S D$ son menores al 8,5\% registrado por Chen et al. (1993) entre un bosque de T. heterophylla y una tala rasa.

La $T S_{15}$ en el $B V$ durante los períodos de crecimiento vegetativo siempre fue menor a la verificada en el $S D$, pero muy parecida a los $2,2^{\circ} \mathrm{C}$ medidos en verano por Carlson y Groot (1997) entre un bosque de $P$. tremuloides y una tala rasa, y mucho menor a los $4,4^{\circ} \mathrm{C}$ señalados por Chen et al. (1993) a $10 \mathrm{~cm}$ de profundidad entre un bosque de T. heterophylla y una tala rasa. Para las $T S_{30}$ las diferencias entre el $B V$ y el $S D$ durante ambos períodos fueron muy similares a los registrados a $15 \mathrm{~cm}$, valores que además son semejantes a los informados por Carlson y Groot (1997).

La $V$ en el $B V$ sólo representó entre el 20 y el $25 \%$ de la observada en el $S D$, valores que se ven altamente afectados cuando se producen ráfagas de viento $\left(V_{e}\right)$ momento en que la velocidad del viento en el $B V$ es alrededor del $40 \%$ de aquella registrada en el exterior (cuadro 1). Estos resultados son consistentes con lo explicado por Lee (1978) y Aussenac $(1997,2000)$, en relación a que las masas boscosas influyen y reducen la velocidad del viento, y que el movimiento de aire dentro del bosque puede ser atribuido a la penetración de remolinos turbulentos a través del follaje. Los registros obtenidos también son respaldados con los trabajos de Chen et al. (1993) en bosques de T. heterophylla, Brosofske et al. (1997) en bosques dominados por Pseudotsuga menziesii (Mirb.) Franco y T. heterophylla al oeste de Washington, cerca de Seattle y Olympia, y Davies-Colley et al. (2000) en un bosque dominado por Beilschmiedia tawa (A. Cunn.) Benth. et Hook.f en el Parque Forestal de Pirongia, Nueva Zelanda ( $\left.38^{\circ} 03^{\prime} \mathrm{S}, 175^{\circ} 04^{\prime} \mathrm{E}\right)$.

Influencia microclimática de la corta de regeneración en un bosque de N. pumilio. Tal como se esperaba, tras la intervención silvicultural de una $C R$, a través de la remoción de aproximadamente un $50 \%$ del área basal del bosque se modificaron las condiciones microclimáticas en el bosque de $N$. pumilio. Estos resultados son consistentes con trabajos similares realizados en otros ecosistemas y diferentes prácticas silviculturales (Marchant 1984, Chen et al. 1993, Otero et al. 1994ab, 1996, 1998, Brosofske et al. 1997, Zheng et al. 2000, Langvall y Örlander 2001, Proe et al. 2001, Holst et al. 2004, Nauertz et al. 2004, Holst y Mayer 2005).

En general, la $R S G$ incidente en la $C R$ fue mayor que en el $B V$ para el período de crecimiento vegetativo 
(cuadro 1), valor cercano a las variaciones de entre 3,0 y 4,5 veces en bosques dominados por Fagus sylvatica L. e intervenidos por medio de cortas de regeneración y localizados en Möhringen, Alemania (Holst y Mayer 2005). También en el período invernal la $R S G$ medida es mayor en la $C R$ que en el $B V$ (cuadro 1). Esta relación supera a la documentada por los mismos autores anteriores (1,3 a 1,5 veces) y a las 1,9 a 2,5 veces calculadas para bosques intervenidos dominados por las especies arbóreas Quercus velutina Lam., Quercus alba L., Quercus coccinea Muenchh., Quercus stellata Wangenh., Carya spp. y Pinus echinata Mill. localizados al sudeste de Missouri Ozarks, Estados Unidos (Zheng et al. 2000). Avalando las tendencias observadas, otras investigaciones han registrado aumentos en la intensidad de la luz incidente en el piso del bosque después de la aplicación de intervenciones silviculturales. Otero et al. (1994a) mencionan aumentos de hasta 1,6, 3,6 y 3,9 veces en la intensidad de la luz (kilolux) a la existente en el interior de un bosque de Nothofagus dombeyi (Mirb.) Oerst., Nothofagus alpina (Poepp. et Endl.) Oerst y Laureolipsis philippiana (Looser) Schoddle, en Panguipulli, Chile, intervenido con cortas en fajas de 30, 60 y $90 \mathrm{~m}$ de ancho. En una corta de protección en claros del dosel (claros de entre 380 y $706 \mathrm{~m}^{2}$ en superficie) en un bosque dominado por Nothofagus nitida (Phil.) Krasser y Drimys winteri J R et Forster la intensidad de la luz difusa aumentó en hasta un promedio de 1,5 veces (Otero et al. 1996, 1998), y hasta 3,2 veces en una corta de regeneración uniforme en un bosque dominado por las especies forestales $N$. dombeyi, $N$. alpina y $N$. pumilio (Otero et al. 1994b).

El comportamiento de $T_{a}, T_{\operatorname{maxa}}$ y $T_{\text {mina }}$ no muestran diferencias significativas entre ambas situaciones estudiadas $(B V$ y $C R$ ) durante los períodos de estudio (crecimiento y receso vegetativo), teniendo un comportamiento muy homogéneo (cuadro 1). Algo similar fue informado por Holst et al. (2004), quienes indican que el impacto de diferentes densidades en el dosel de un bosque de $F$. sylvatica, producto de la aplicación de cortas de regeneración, no provocaría grandes diferencias en el comportamiento de la temperatura media mensual del aire. Esta tendencia también es determinada por Nauertz et al. (2004), en intervenciones de bosques dominados por Acer saccharum Marsh en el Bosque Nacional de Ottawa, Península Superior de Michigan en Estados Unidos. Otero et al. $(1996,1998)$ señalan que las temperaturas promedio del aire durante el día tienden a ser superiores en $1^{\circ} \mathrm{C}$ dentro de las áreas intervenidas a través de sistema de cortas de protección en claros respecto al bosque virgen de $N$. nitida - $D$. winteri. Otero et al. (1994a) comentan que la $T_{a}$ se ve incrementada $1,1,1,3$ y 1,4 veces producto de las cortas en fajas de 30, 60 y $90 \mathrm{~m}$ en un bosque de $N$. dombeyi $N$. alpina - L. philippiana, respectivamente; mientras que en una corta de regeneración homogénea en un bosque dominado por $N$. dombeyi - N. alpina - N. pumilio la $T_{a}$ aumenta $3^{\circ} \mathrm{C}$ (Otero et al. 1994b). Sin embargo, respecto a $T_{\text {mina }}$ el $B V$ tiene un comportamiento intermedio entre $S D$ y $C R$ durante el verano. $T_{\text {mina }}$ en $C R$ puede ser más alta que en $B V$ producto de que en $C R$, al tener una menor cobertura de dosel, recibe mayores montos de radiación solar durante el día. Por otro lado, en SD, al no presentar cobertura arbórea, la pérdida de radiación neta de onda larga se ve incrementada en la noche, lo que probablemente produciría menores temperaturas diarias promedio (Holst et al. 2004).

La $H R_{a}$ en la $C R$ tuvo un comportamiento promedio diferente a lo observado por Aussenac (1997), quien indica que esta variable debería manifestar valores entre los registrados en un rodal sin intervención y una tala rasa. Esto se puede deber a que el viento es más fuerte en $S D$, mezclando el aire, lo cual transporta energía calórica y humedad y por lo tanto reduce la variación de su humedad (Chen et al. 1993). Además, en $C R$ el viento es más débil y estable, permitiendo humedades del aire más extremas (Chen et al. 1993). En este estudio la $H R_{a}$ en la $C R$ fue menor a la registrada en $B V$ (en ambos períodos de crecimiento y receso vegetativo), situación muy parecida a lo señalado por Otero et al. $(1996,1998)$, quienes demostraron fluctuaciones hasta $8 \%$ menores en una corta de protección en claros de bosque (protección en grupos), disminuciones de entre 3,9 y $9,8 \%$ en cortas en fajas de 60 y $90 \mathrm{~m}$ de ancho (Otero et al. 1994a), y de en promedio un $9 \%$ para una corta de regeneración homogénea (Otero et al. 1994b).

Las $T S_{15}$ y $T S_{30}$, aunque siempre fueron mayores en la $C R$ durante el período de crecimiento vegetativo, no mostraron diferencias significativas con el $B V$ (cuadro 1), lo cual puede deberse a una posible mayor acumulación de nieve en $C R$ que en $B V$. En cambio, durante el período sin follaje, el $B V$ presentó mayores temperaturas, siendo estadísticamente diferente a los $30 \mathrm{~cm}$ de profundidad (cuadro 1). Resultados similares son mostrados en estudios realizados por Holst et al. (2004), en que cortas de regeneración en bosques dominados por $F$. sylvatica no mostrarían influencia en estas variables. Sin embargo, aunque en una faja de $60 \mathrm{~m}$ en un bosque de $N$. dombeyi - N. alpina - L. philippiana la temperatura del suelo a $10 \mathrm{~cm}$ de profundidad aumentó 2,1 veces respecto a la registrada en el bosque virgen, en una faja de $90 \mathrm{~m}$ esta variable es $3^{\circ} \mathrm{C}$ superior que aquellas registradas en la faja de $60 \mathrm{~m}$ (Otero et al. 1994a). Al mismo tiempo, aumentos de hasta en promedio dos veces en la temperatura del suelo a $10 \mathrm{~cm}$ de profundidad fueron registrados en una corta de regeneración homogénea en un bosque dominado por $N$. dombeyi - N. alpina - N. pumilio (Otero et al. 1994b).

La $V$ en promedio fue estadísticamente mayor en la $C R$ que en el $B V$ (cuadro 1), pues a menor densidad del bosque se favorece la penetración del viento y las turbulencias, pudiendo llegar a producirse caída de árboles si sus raíces no se han adaptado a las nuevas condiciones (Aussenac 1997). En esta investigación no se encontraron diferencias en las $V_{e}$ medidas en ambos bosques (cuadro 1), así el 
sector intervenido, con una menor densidad de árboles, no provocaría aumentos para esta variable.

\section{CONCLUSIONES}

Las tendencias observadas en las variables meteorológicas medias se ajustan al comportamiento que éstas normalmente exhiben en bosques de similares características. Se pudieron detectar pequeñas diferencias en las magnitudes de sus montos atribuidas a las condiciones climáticas específicas y a la ubicación geográfica de los sectores evaluados.

$\mathrm{Al}$ intervenirse el bosque virgen con una corta de regeneración y producto de la consiguiente alteración de la estructura del dosel, se modifica el microclima del bosque de $N$. pumilio. La corta de regeneración produce incrementos significativos de la radiación solar global incidente y de la velocidad media del viento y una disminución significativa de la humedad relativa del aire. No se verifican variaciones estadísticamente significativas en las magnitudes de las temperaturas del aire, del suelo y de la velocidad media de las extremas del viento al comparar ambos bosques. Sin embargo, al abrir el dosel en la corta de regeneración la velocidad del viento es más alta y se prolonga durante todo el año, lo que en definitiva provoca que los árboles estén más susceptibles a caerse o quebrarse por el efecto de un aumento intempestivo en la velocidad del viento.

Por lo tanto, estos resultados sugieren que las diferencias en las variables microclimáticas dependerían de las variaciones estructurales entre rodales, para bosques con similares estados de desarrollo y localizados en la misma posición topográfica y geográfica. La modificación del microclima del bosque producto de la aplicación de la corta de regeneración cambia las condiciones del sitio y por consiguiente influye en los procesos ecológicos, tales como la composición de especies, el establecimiento, el crecimiento y la mortalidad de plantas.

\section{AGRADECIMIENTOS}

El estudio fue financiado con aportes del Fondo Nacional de Investigaciones Científicas y Tecnológicas (FONDECYT) a través de los Proyectos No 1960936 y 1000645 , y del Gobierno Regional de Magallanes y Antártica Chilena (Proyecto FNDR "Seguimiento Forestal y Ambiental del Uso de los Bosques de Lenga - XII Región”). Al mismo tiempo agradecemos el apoyo logístico y el trabajo de campo realizado por el Ingeniero Forestal Marcelo Álvarez y otros colaboradores de la Corporación Nacional Forestal.

\section{REFERENCIAS}

Aussenac G. 1997. Interactions between climates and forests at the local, regional, and global levels. In Birot $\mathrm{Y}$ ed.
Sustainable forest management: Contribution of research Zvolen, Slovakia. Arbora Publishers spol. s.r.o. p. 1-16. (IUFRO Occasional Paper $N^{\circ}$ 9).

Aussenac G. 2000. Interactions between forest stands and microclimate: Ecophysiological aspects and consequences for silviculture. Annals of Forest Science 57: 287-301.

Barnes BV, DR Zak, SR Denton, SH Spurr. 1998. Forest ecology. 4 ed. New York, USA. John Wiley. 774 p.

Bartsch N, C Rapp. 1995. Regeneración de la lenga (Nothofagus pumilio) en una tala rasa en hueco. Investigación en el bosque de montaña andino-patagónico en Argentina. In Regeneración Natural de la Lenga. Factores Ecológicos. Esquel, Argentina. CIEFAP. p. 49-73. (Publicación Técnica No 21).

Brosofske KD, J Chen, RJ Naiman, JF Franklin. 1997. Harvesting effects on microclimatic gradients from small streams to uplands in Western Washington. Ecological Applications 7: 1188-1200.

Caldentey J, A Promis, H Schmidt, M Ibarra. 1999-2000. Variación microclimática causada por una corta de protección en un bosque de lenga (Nothofagus pumilio). Ciencias Forestales 14: $51-59$.

Caldentey J, M Ibarra, J Hernández. 2001. Litter fluxes and decomposition in Nothofagus pumilio stands in the region of Magallanes, Chile. Forest Ecology and Management 148: 145-157.

Caldentey J, H Mayer, M Ibarra, A Promis. 2009. The effects of a regeneration felling on photosynthetic photon flux density and regeneration growth in a Nothofagus pumilio forest. European Journal of Forest Research 128: 75-84.

Carlson DW, A Groot. 1997. Microclimate of clear-cut, forest interior, and small openings in trembling aspen forest. Agricultural and Forest Meteorology 87: 313-329.

Chen J, JF Franklin, TA Spies. 1993. Contrasting microclimates among clearcut, edge, and interior of old-growth Douglas-fir forest. Agricultural and Forest Meteorology 63: 219-237.

Chen J, SC Saunders, TR Crow, RJ Naiman, KD Brosofske, GD Mroz, BL Brookshire, JF Franklin. 1999. Microclimate in forest ecosystems and landscape ecology. Variations in local climate can be used to monitor and compare the effects of different management regimes. BioScience 49(4): 288-297.

CONAF (Corporación Nacional Forestal, CL), CONAMA (Comisión Nacional del Medio Ambiente, CL), BIRF (Banco Internacional de Reconstrucción y Fomento, USA), Universidad Austral de Chile, Pontificia Universidad Católica de Chile, Universidad Católica de Temuco. 1999. Proyecto catastro y evaluación de recursos vegetacionales nativos de Chile. Informe Regional Duodécima Región. Santiago, Chile. CONAF. 93 p.

Contreras H, R Borgel, M Quezada, V García de Cortázar, M Rojas, W Bitterlich. 1975. Informe de la primera etapa del proyecto sobre reforestación de la Precordillera Patagónica (Cuadrángulos Skyring y Rubens). Santiago, Chile. Facultad de Ciencias Forestales, Universidad de Chile. 76 p.

Crow TR, DS Buckley, EA Nauertz, JC Zasada. 2002. Effects of management on the composition and structure of northern hardwood forests in Upper Michigan. Forest Science 48: 129-145.

Davies-Colley RJ, GW Payne, M van Elswijk. 2000. Microclimate gradients across a forest edge. New Zealand Journal of Ecology 24(2): 111-121. 
Decocq G, M Aubert, F Dupont, J Bardat, A Wattez-Franger, R Saguez, B de Foucault, D Alard, A Delelis-Dusollier. 2005. Silviculture-driven vegetation change in a European temperate deciduous forest. Annals of Forest Science 62: 313-323.

Deferrari G, C Camilion, G Martínez Pastur, P Peri. 2001. Changes in Nothofagus pumilio forest biodiversity during the forest management cycle: 2. Birds. Biodiversity and Conservation 10: 2093-2108.

Doberti H, E Ruz. 1981. Aspectos climáticos de las distintas zonas ecológicas de uso ganadero en la XII Región. Agricultura Técnica 41: 153-163.

Frangi J, L Richter. 1994. Balances hídricos de bosques de Nothofagus en Tierra del Fuego, Argentina. Revista de la Facultad de Agronomía (La Plata) 70: 65-79.

Gajardo R. 1994. La vegetación natural de Chile. Clasificación y distribución geográfica. Santiago, Chile. Editorial Universitaria. $165 \mathrm{p}$.

Gea-Izquierdo G, G Martínez Pastur, JM Cellini, MV Lencinas. 2004. Forty years of silvicultural management in southern Nothofagus pumilio primary forests. Forest Ecology and Management 201: 335-347.

Geiger R, RH Aron, P Todhunter. 2003. The climate near the ground. 6 ed. Lanham, USA. Rowman \& Littlefield Publishers. 584 p.

Ghuman BS, R Lal. 1987. Effects of partial clearing on microclimate in a humid tropical forest. Agricultural and Forest Meteorology 40: 17-29.

Holst T, H Mayer. 2005. Radiation components of beech stands in Southwest Germany. Meteorologische Zeitschrift 14(2): 107-115.

Holst T, H Mayer, D Schindler. 2004. Microclimate within beech stands - part II: thermal conditions. European Journal of Forest Research 123: 13-28.

INIA (Instituto de Investigaciones Agropecuarias, CL). 1989. Mapa agroclimático de Chile. Santiago, Chile. INIA. 221 p.

Lara A, G Cruz. 1987. Vegetación del área de uso agropecuario de la XII Región, Magallanes y la Antártica Chilena. Santiago, Chile. INIA. 23 p.

Langvall O, G Örlander. 2001. Effects of pine shelterwoods on microclimate and frost damage to Norway spruce seedlings. Canadian Journal of Forest Research 31: 155-164.

Lee R. 1978. Forest microclimatology. New York, USA. Columbia University Press. 276 p.

Lencinas MV, G Martínez Pastur, M Medina, C Busso. 2005. Richness and density of birds in timber Nothofagus pumilio forests and their unproductive associated environments. Biodiversity and Conservation 14: 2299-2320.

Loguercio GA. 1995. Crecimiento de la regeneración de lenga (Nothofagus pumilio (Poepp et Endl.) Krasser) y su dependencia de las condiciones dominantes de radiación. In Regeneración Natural de la Lenga. Factores Ecológicos. Esquel, Argentina. CIEFAP. p. 1-47. (Publicación Técnica No 21).

Marchant HA. 1984. Estudio micrometeorológico en un bosque siempreverde intervenido con distintos métodos de repoblación. Tesis Ingeniero Forestal. Valdivia, Chile. Facultad de Ciencias Forestales, Universidad Austral de Chile. 139 p.

Martínez Pastur G, PL Peri, MC Fernández, G Staffieri. 1999. Desarrollo de la regeneración a lo largo del ciclo del manejo forestal de un bosque de Nothofagus pumilio: 1 . Incidencia de la cobertura y el aprovechamiento o cosecha. Bosque 20: 39-46.
Martínez Pastur G, JM Cellini, PL Peri, RF Vukasovic, MC Fernández. 2000. Timber production of Nothofagus pumilio forests by a shelterwood system in Tierra del Fuego (Argentina). Forest Ecology and Management 134: 153-162.

Martínez Pastur G, PL Peri, MC Fernández, G Staffieri, MV Lencinas. 2002. Changes in understory species diversity during the Nothofagus pumilio forest management cycle. Journal of Forest Research 7: 165-174.

Nauertz EA, TR Crow, JC Zasada, RM Teclaw. 2004. Microclimatic variation between managed and unmanaged northern hardwood forests in Upper Michigan, USA. Saint Paul, USA. U.S. Department of Agriculture, Forest Service, North Central Research Station. 31 p. (Gen. Tech. Rep. NC-236).

Otero L, A Contreras, L Barrales. 1994a. Efectos ambientales de diferentes tipos de corta en bosque nativo. El caso de las cortas de protección en fajas. Ciencia e Investigación Forestal 8: 87-118.

Otero L, P Donoso, L Barrales. 1994b. Efectos ambientales de las cortas de protección en bosque nativo. In Actas Seminario medio ambiente, biodiversidad y actividades productivas. Santiago, Chile. INFOR. p. 301-321.

Otero L, A Contreras, L Barrales. 1996. Cortas de protección en hoyos de luz: efectos microclimáticos. Ciencia e Investigación Forestal 10: 277-288.

Otero L, A Contreras, L Barrales. 1998. Cortas de protección en claros de bosque: Efectos microclimáticos. In Actas Primer Congreso Latinoamericano IUFRO. Valdivia, Chile. 8 p.

Pisano E. 1977. Fitogeografía de Fuego-Patagonia chilena. I.- Comunidades vegetales entre las latitudes $52^{\circ}$ y $56^{\circ} \mathrm{S}$. Anales del Instituto de la Patagonia 8: 121-250.

Pisano E. 1981. Bosquejo fitogeográfico de Fuego-Patagonia. Anales del Instituto de la Patagonia 12: 159-171.

Proe MF, JH Griffiths, HM McKay. 2001. Effect of whole-tree harvesting on microclimate during establishment of second rotation forestry. Agricultural and Forest Meteorology 110: 141-154.

Reifsnyder WE, GM Furnival, JL Horowitz. 1971/1972. Spatial and temporal distribution of solar radiation beneath forest canopies. Agricultural Meteorology 9: 21-37.

Roig FA, J Anchorena, O Dollenz, AM Faggi, E Méndez. 1985. Las comunidades vegetales de la Transecta Botánica de la Patagonia Austral. In Boelcke O, DM Moore, FA Roig eds. Transecta Botánica de la Patagonia Austral. Buenos Aires, Argentina. Conicet (Argentina), Royal Society (Gran Bretaña), Instituto de la Patagonia (Chile). p. 350-519.

Rosenfeld JM, RM Navarro Cerrillo, JR Guzmán Álvarez. 2006. Regeneration of Nothofagus pumilio [Poepp. et Endl.] Krasser forests after five years of seed tree cutting. Journal of Environmental Management 78: 44-51.

Schmidt H. 1994. Posibilidades de uso sustentable de los bosques de lenga: El caso de Magallanes - Chile. In Montes L, G Oliva eds. Patagonia. Actas del taller internacional sobre recursos fitogenéticos, desertificación y uso sustentable. Río Gallegos, Argentina. INTA. p. 128-137.

Schmidt H, A Urzúa. 1982. Transformación y manejo de los bosques de lenga en Magallanes. Ciencias Agrícolas $N^{\circ} 11$. Santiago, Chile. Facultad de Ciencias Agrarias, Veterinarias y Forestales, Universidad de Chile. 62 p.

Schmidt H, G Cruz, A Promis, M Álvarez. 2003. Transformación de los bosques de lenga vírgenes e intervenidos a bosques 
manejados. Guía para los bosques demostrativos. Publicaciones Misceláneas $N^{\circ}$ 4. Santiago, Chile. Facultad de Ciencias Forestales, Universidad de Chile. 60 p.

Skottsberg C. 1910. Botanische Ergebnisse der Schwedischen Expedition nach Patagonien und dem Feuerlande 1907-1909. I. Übersicht über die wichtigsten Pflanzenformationen Südamerikas S. von $41^{\circ}$, ihre geographische Verbreitung zum Klima. Kungl. Svenske Vtenskapsakademiens Handlingar 46(3): 1-28.

Spagarino C, G Martínez Pastur, P Peri. 2001. Changes in Nothofagus pumilio forest biodiversity during the forest management cycle: 1. Insects. Biodiversity and Conservation 10: 2077-2092.

Vergara PM, RP Schlatter. 2006. Aggregate retention in two Tierra del Fuego Nothofagus forests: Short-term effects on bird abundance. Forest Ecology and Management 225: 213-224.

Venegas C, RP Schlatter. 1999. Efecto de la intervención silvícola en bosques de Nothofagus pumilio sobre ensambles avifaunísticos estivales en Tierra del Fuego (Chile). Anales del Instituto de la Patagonia 27: 41-50.

Zheng D, J Chen, B Song, M Xu, P Sneed, R Jensen. 2000. Effects of silvicultural treatments on summer forest microclimate in southeastern Missouri Ozarks. Climate Research 15: 45-59.

Recibido: 16.09 .09

Aceptado: 15.03.10 